\title{
Efectos de la invasión del ligustro, Ligustrum lucidum, en la dinámica hídrica de las Yungas del noroeste Argentino
}

\author{
Effects of glossy privet’s invasion on the water dynamics of the Argentinean Yungas forest \\ Lucía Zamora Nasca a*, Lia Montti a,b,c, Ricardo Grau a,b, Leonardo Paolini a,b \\ "Autor de correspondencia: a Universidad Nacional de Tucumán, Instituto de Ecología Regional (IER), CC 34, \\ Yerba Buena, Tucumán, Argentina, tel.: 54 (381) 4255174, luciabzamora@hotmail.com \\ ${ }^{\mathrm{b}}$ Consejo Nacional de Investigaciones Científicas y Técnicas (CONICET), Argentina. \\ ${ }^{\mathrm{c} C e n t r o ~ d e ~ I n v e s t i g a c i o n e s ~ d e l ~ B o s q u e ~ A t l a ́ n t i c o ~(C e I B A), ~ M i s i o n e s, ~ A r g e n t i n a . ~}$
}

\begin{abstract}
SUMMARY
Argentinean Yungas (subtropical montane forests) have high biodiversity and play a key role in regional watershed regulation. Ligustrum lucidum (glossy privet), native of China, is one of the dominant exotic tree species in secondary forests of this ecoregion. Due to its high growth rates and green foliage throughout the year -in contrast to native forests which are partially deciduous- the hypothesis is that water availability is reduced in glossy privet forests, especially during dry season (winter-spring). Six pairs of plots both in native and secondary glossy privet dominated forests in Sierra San Javier, Tucumán, Argentina were sampled. Soil moisture, by measuring both volumetric water content and water potential, and estimated productivity of different types of forests, through normalized difference vegetation index (NDVI derived from satellite imagery), were monitored. During 2011 - 2012 dry seasons, soil volumetric water content in glossy privet forests was $32.5 \%$ and $24 \%$ lower than in native forests; while soil water potential was respectively eight and four times lower. In glossy privet forests NDVI values were $8.5 \%$ higher than in native forests; particularly in lower altitude forests and those with a higher winter-spring water deficit. These results suggest that the glossy privet forest would be consuming more water, especially during the dry season, due to more prolonged phenology activity compared to native forests. In consequence, glossy privet invasion in Argentinean Yungas could alter water functioning of this ecosystem.
\end{abstract}

Key words: forest-phenology, invasion by exotic trees, MODIS, NDVI, water consumption.

\section{RESUMEN}

Las Yungas argentinas (bosques de montaña) tienen alta biodiversidad y un rol clave en la regulación hídrica. El ligustro (Ligustrum lucidum) es una de las especies exóticas que dominan sus bosques secundarios. Debido a sus altas tasas de crecimiento y follaje verde todo el año, se planteó la hipótesis de que la disponibilidad de agua en el suelo se reduciría en bosques de ligustro en comparación con bosques nativos, principalmente durante la época seca (invierno-primavera). Se trabajó en seis pares de parcelas de bosques nativos y bosques secundarios monodominantes de ligustro en la Sierra San Javier, Tucumán, Argentina. Se registró la humedad edáfica mediante mediciones de contenido volumétrico y potencial hídrico y se estimó la productividad de los distintos tipos de bosque mediante el índice de vegetación de diferencia normalizada (NDVI). Durante la época seca de 2011 y 2012, los suelos en ligustrales presentaron un contenido volumétrico de agua $32,5 \%$ y $24 \%$ y un potencial hídrico en el suelo ocho y cuatro veces menor. Se observaron mayores valores de NDVI (8,5 \%) en bosques de ligustro que en bosques nativos, principalmente en las selvas localizadas a menor altura y con mayor déficit hídrico inverno-primaveral. Los resultados sugieren que el bosque de ligustro estaría consumiendo mayor cantidad de agua, sobre todo en la época seca, debido a una actividad fenológica más prolongada respecto al bosque nativo y que su invasión en las Yungas podría alterar el funcionamiento hídrico de este ecosistema.

Palabras clave: consumo de agua, fenología, invasión por árboles exóticos, MODIS, NDVI.

\section{INTRODUCCIÓN}

Los sistemas naturales y sociales dependen de la disponibilidad de agua, que además interviene en el intercambio de energía con la atmósfera y en el ciclo de nutrientes. Los cambios en la vegetación asociados a cambios climáticos y a actividades humanas modifican el movimiento del agua afectando el consumo y la conexión entre los distintos componentes del ciclo hidrológico (Nosetto et al. 2011). Entre los componentes principales del cambio ambiental con po- tencial efecto sobre los ciclos hidrológicos, se destacan el cambio climático, el cambio de uso del suelo y la invasión por especies exóticas (Mooney y Hobbs 2000). Esta última puede alterar las funciones ecosistémicas a escala local y regional con importantes consecuencias ecológicas y económicas (Mack et al. 2000). Las plantas invasoras presentan altas tasas de crecimiento y reproducción, gran capacidad de aclimatación y patrones fenológicos diferentes a las especies nativas (Tecco et al. 2010). Tales características frecuentemente resultan en una mayor productividad, lo que favore- 
ce su desplazamiento por exclusión competitiva y ocasiona cambios en la utilización de los recursos, principalmente agua y nutrientes del suelo; y cambios ecológicos que potencialmente pueden afectar la biodiversidad (Mark 2009).

Actualmente, las invasiones de leñosas exóticas son una preocupación mundial debido a su impacto sobre la biodiversidad y los servicios ecosistémicos. Le Maitre et al. (1996) documentaron reducciones significativas en los caudales de cuencas asociadas a especies exóticas invasoras (ejemplo: Acacia mearnsii De Wild.) y a plantaciones forestales exóticas de rápido crecimiento (ejemplo: Eucalyptus spp. y Pinus spp.). Estos autores, modelaron para Ciudad del Cabo, Sudáfrica (clima mediterráneo) las consecuencias de la invasión de plantas exóticas en áreas de captación de agua y estimaron una pérdida del $30 \%$ del agua suministrada anualmente debido a las altas tasas de evapotranspiración de dichas especies. En el oeste de Norteamérica, Tamarix spp., arbusto invasor, ha colonizado cientos de hectáreas de llanuras aluviales, ambientes riparios y otros humedales, disminuyendo los flujos de agua cercanos (Shafroth et al. 2005). A su vez, estudios en Uruguay (Silveira et al. 2006) y Brasil (Paula Lima 2010) demostraron que las forestaciones de exóticas afectaban la escorrentía, los caudales pico, volúmenes escurridos y el contenido de agua edáfica.

Las Yungas Argentinas son bosques subtropicales de montaña que presentan alta biodiversidad en relación a otros biomas de Argentina y juegan un rol clave en la provisión de servicios ecosistémicos. En Tucumán, constituyen el ecosistema con mayores precipitaciones $y$, dado su balance hídrico positivo, son el principal lugar de captación de los numerosos ríos que abastecen el grueso de las agroindustrias regionales (caña de azúcar, citrus, horticultura) y aproximadamente a un millón y medio de personas. Estos bosques poseen un fuerte gradiente altitudinal y ambiental por lo que la vegetación se organiza en pisos de características fisonómicas y florísticas diferenciables: Selva Pedemontana, Selva Montana y Bosque Montano (Brown 2009). El piedemonte de las Yungas presenta un sostenido aumento de la población urbana y una progresiva intensificación agrícola en las décadas recientes, mientras que en las zonas de montaña prevalecen los procesos de abandono de tierras agrícolas como consecuencia de cambios socioeconómicos y dificultades para la mecanización de la agricultura y la conservación de suelos (Grau et al. 2008). Sobre estas áreas de abandono agrícola, en las últimas décadas se observa la expansión de bosques secundarios, muchos de ellos dominados por especies exóticas (Grau y Aragón 2000). Este proceso podría afectar el funcionamiento de este ecosistema, específicamente la transferencia de agua entre suelo, napa freática, vegetación y atmósfera.

Entre las especies exóticas de las Yungas que dominan los bosques secundarios se destaca el ligustro o siempreverde (Ligustrum lucidum W.T. Aiton, Oleaceae) (Aragón y Morales 2003). Esta especie, originaria de China, presenta numerosas características que la convierten en una colonizadora exitosa y en un factor de cambios ambien- tales potencialmente intensos en las próximas décadas. Estudios previos (Casavecchia 2004) a partir de imágenes satelitales en la Sierra de San Javier sugieren diferencias en la fenología y productividad de los bosques invadidos por ligustro respecto a los nativos ya que en la época seca los bosques de ligustro presentan valores de reflectancia que se reflejarían en valores mayores de NDVI (índice de vegetación de diferencia normalizada, índice indirecto de actividad fotosintética basado en datos satelitales) que los bosques nativos cercanos. Paralelamente, estudios fisiológicos demuestran que en especies arbóreas subtropicales existe una correlación positiva entre la tasa de crecimiento, la fotosíntesis y el consumo de agua (Campanello 2004). Este hecho, sumado a las altas tasas de crecimiento del ligustro (Lichstein et al. 2004, Easdale et al. 2007), sugiere que los bosques de esta especie estarían efectuando un mayor consumo de agua que los bosques nativos.

El ligustro ha invadido numerosas regiones del mundo (Cronk y Fuller 1995). En Argentina fue introducido a principios de siglo (Río y Achaval 1904), utilizado principalmente como ornamental y en cercos vivos (Panetta 2000), siendo el primer ejemplar de herbario colectado por Cabrera en 1932 (Instituto de Botánica Darwinion). Se convirtió en una invasora importante en Yungas del noroeste del país, sierras de Córdoba y en la provincia de Buenos Aires (Montaldo 2000, Aragón y Morales 2003, Gavier-Pizarro et al. 2012). Particularmente, la invasión del ligustro podría tener importantes efectos en la dinámica hídrica de las Yungas, especialmente en la estación de mayor déficit hídrico (julio-setiembre). Por esto, en este trabajo se plantea la hipótesis de que, principalmente durante la época seca (invierno-primavera), la disponibilidad de agua en suelo disminuye en los bosques dominados por ligustro en comparación con bosques nativos. Para evaluar estos efectos, se consideran los siguientes objetivos: 1) comparar el contenido de agua edáfica y su disponibilidad para la planta (potencial hídrico) en bosques dominados por ligustro y en bosques nativos a lo largo de un año; y 2) analizar la variación estacional del índice de vegetación de diferencia normalizada (NDVI), derivado de imágenes satelitales, como indicador de la productividad primaria y la actividad fenológica de ambos bosques, relacionando esta variable con las condiciones climáticas anuales.

\section{MÉTODOS}

Área de estudio. Se trabajó en la ecorregión de las Yungas Argentinas en la zona cumbral y vertiente oriental de la Sierra San Javier, $15 \mathrm{~km}$ al oeste de la ciudad de San Miguel de Tucumán, Argentina (27 $30^{\prime} \mathrm{S}, 65^{\circ} 40^{\prime} \mathrm{O}$ ) (figura 1), en dos niveles altitudinales de la Selva Montana: (1) el límite inferior de la Selva Basal, entre los 630 y 780 m snm y (2) el sector de Selva de Mirtáceas, entre 1.280 y 1.450 m snm. El primero se caracteriza por presentar un mayor porcentaje de especies caducifolias, debido a su mayor estacionalidad hídrica: el 90 \% de los 1.200 mm anuales de las precipitaciones ocurren en la temporada cálida y presenta un déficit hí- 


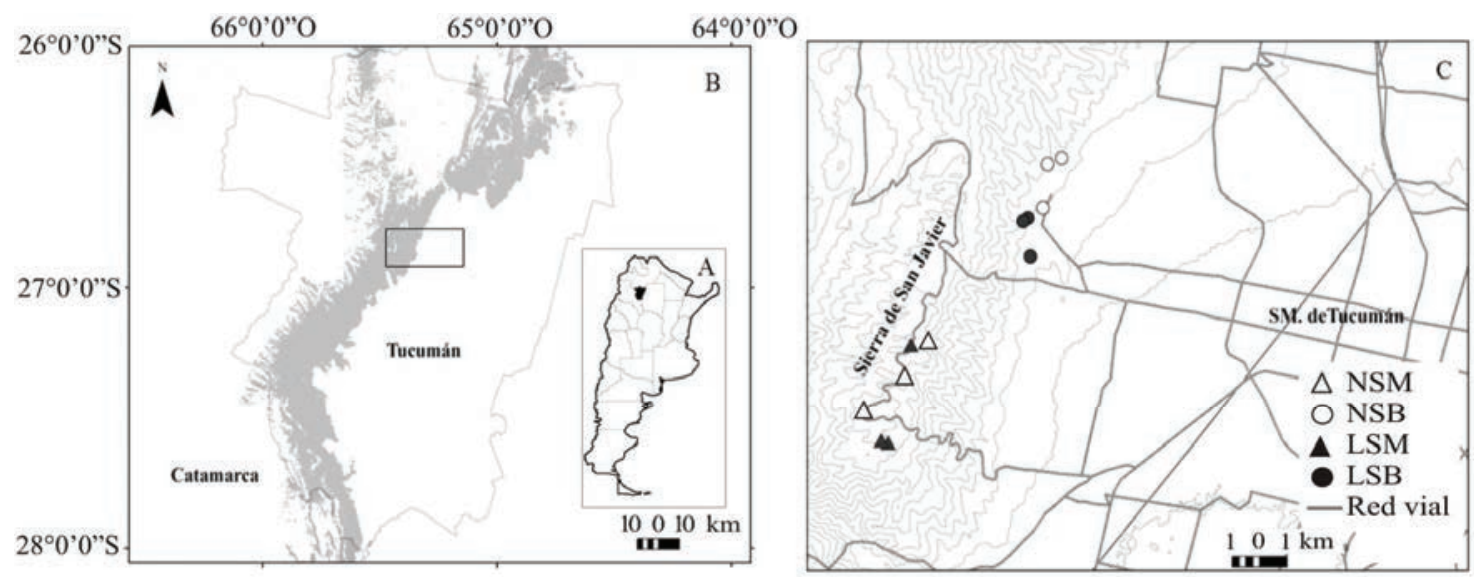

Figura 1. Mapa del área de estudio. A) Ubicación de la provincia de Tucumán en Argentina (negro). B) Provincia de Tucumán y detalle del área de estudio. C) Áreas donde se encuentran las parcelas de bosques nativos en ambos niveles altitudinales, Selva Basal (O NSB) y Selva de Mirtáceas ( $\triangle$ NSM) y los parches de bosques invadidos por ligustro en ambos niveles altitudinales, Selva Basal (• LSB) y Selva de Mirtáceas ( $\boldsymbol{\Delta}$ LSM).

Study area. A) Location of Tucumán in Argentina (black). B) Province of Tucumán and detail of the study area. C) Areas where native forest patches are placed in both altitudinal levels, Basal Forest ( $\circ$ NSB) and Myrtaceae Forest ( $\triangle$ NSM) and forest patches invaded by glossy privet in both altitudinal levels, Basal Forest $(\bullet$ LSB $)$ and Myrtaceae Forest $(\boldsymbol{\Delta}$ LSM $)$.

drico en el período invernal-primaveral, con precipitaciones a veces inferiores a los $10 \mathrm{~mm}$ mensuales y sin aportes adicionales de neblina. La temperatura media en invierno oscila entre 10 y $12{ }^{\circ} \mathrm{C}$ y en verano entre 24 y $26^{\circ} \mathrm{C}$. El sector de Selva de Mirtáceas es más frío, con predominio de especies perennifolias, posee menor estacionalidad hídrica y representa la franja altitudinal de máximas precipitaciones pluviales (más de $1.500 \mathrm{~mm}$ anuales); donde las neblinas hacen un aporte importante durante el invierno (Hunzinger 1997).

Ambos sectores altitudinales fueron deforestados para explotaciones agrícolas que luego fueron abandonadas, encontrándose actualmente cientos de hectáreas de bosques en estado de sucesión secundaria, monodominados por ligustro (Grau et al. 2008). Estos bosques presentan mayor área basal y biomasa acumulada que sus coetáneos nativos, como consecuencia de poseer la mayor tasa de crecimiento observada para árboles de las Yungas (Easdale et al. 2007), pero presentan menor biodiversidad animal y vegetal (Ayup et al. 2014). El ligustro presenta un desfasaje fenológico con respecto a las especies nativas (Grau y Aragón 2000), altísima producción de frutos durante el invierno, eficiente dispersión de semillas por aves, buena capacidad de reproducción vegetativa y germinación y supervivencia en un amplio rango de hábitats (Aragón y Groom 2003).

Diseño experimental. Se trabajó en cinco y seis pares de unidades de bosques dominados por ligustro (L) y por bosques nativos (N) según el objetivo. Tres pares en Selva de Mirtáceas (SM) y tres en Selva Basal (SB). Dichos pares presentaron similares condiciones de pendiente, exposición, altitud y edad sucesional, cubriendo un rango de edades de entre 15 y 40 años. Los suelos presentan gran variabilidad debido a la topografía, pero en general son tipo franco y franco arenoso, del orden Molisoles, con contenidos de nitrógeno y fósforo de 0,26 a 0,73 y 7,2 a 31,2, respectivamente $^{1}$. En general, en la Selva de Mirtáceas se encuentran suelos formados a partir de materiales originarios diferentes: los desarrollados a partir de limos loésicos, en la mayor parte del área cumbral, y los formados a partir de materiales meteorizados de edad Cretácica (Formación El Cadillal), mezclados con material limo loésico y clastos del basamento metamórfico. Su característica general es el desarrollo edáfico y el importante contenido de materia orgánica en los horizontes superficiales. En la Selva Basal, predominan los suelos formados a partir de una cubierta loésica más potente, retransportada y por material clástico, dando lugar a suelos aptos para la agricultura (Sesma et al. 2010). El perfil varía con la ubicación altitudinal pero en general presenta un horizonte A de profundidad variable entre los 0 a $50 \mathrm{~cm}$, negro a pardo muy oscuro, franco con gravas y guijarros, muy friable, ligeramente adhesivo y plástico y un horizonte $C$ entre los $50 \mathrm{~cm}$ y los $170 \mathrm{~cm}$ de profundidad de gravas y guijarros (Zuccardi et al. 1967). Los pares de sitios estuvieron separados por no menos de $2 \mathrm{~km}$ para garantizar la independencia de las muestras (figura 1C).

Para cumplir con el objetivo 1, a lo largo del año, en cinco pares de unidades de bosques (invadido por ligustro y nativo) se registraron: a) contenido de humedad edáfica y $b$ ) potencial hídrico del suelo. Para llevar a cabo el objetivo 2 se identificaron seis parcelas de gran tamaño de cada tipo de bosque y se obtuvo el NDVI a partir de imágenes satelitales. A continuación se detalla cada etapa de la metodología.

\footnotetext{
María Marta Ayup. Universidad Nacional de Tucumán, Instituto de Ecología Regional (IER), Yerba Buena, Tucumán, Argentina. Datos no publicados.
} 
En los bosques nublados de montaña las napas freáticas no constituyen una fuente significativa de agua para la vegetación ya que las mismas se encuentran por debajo de los 20 a 30 metros de profundidad (Tineo 1985, Eremchuk y Martensen 1996), siendo la neblina y las lluvias el aporte principal de agua (Hunzinger 1997). Por esto, para determinar la diferencia de humedad edáfica (contenido volumétrico $\left(\theta_{\mathrm{v}}\right)$ y potencial hídrico) entre ambos bosques (objetivo 1) en cinco pares se tomó una vez al mes, entre julio 2011 y setiembre 2012, mediante el uso de barreno, tres muestras de suelo a $25 \mathrm{~cm}$ de profundidad, separadas por una distancia mínima de $15 \mathrm{~m}$ entre sí. Se guardaron en bolsas de plástico herméticas y se llevaron a laboratorio para su procesamiento. Dado el alto contenido de grava y guijarros de los suelos, esta profundidad de muestreo se consideró un buen compromiso entre el esfuerzo de cavado y la posibilidad de obtener medidas de humedad en la zona de mayor densidad radicular.

Para evaluar el contenido volumétrico $\left(\theta_{\mathrm{v}}\right)$ de agua en suelo, que expresa el contenido de agua en relación a un volumen de suelo, se extrajo una muestra de volumen conocido y se la comparó con su peso húmedo y su peso luego de secarse a $105^{\circ} \mathrm{C}$ hasta peso constante, a base de la fórmula [1]:

$\theta_{\mathrm{v}}=\omega_{\mathrm{g}} \times \mathrm{DA}$

Donde,

$\theta_{\mathrm{v}}=$ contenido volumétrico de agua $\left(\mathrm{mL} \mathrm{cm}^{-3}\right)$.

$\omega_{\mathrm{g}}=$ contenido gravimétrico de agua $\left(\mathrm{g} \mathrm{g}^{-1}\right.$ suelo seco $)=$ [(suelo húmedo (g) - suelo seco (g))/( suelo seco (g))]*100. $\mathrm{DA}=$ densidad aparente del suelo $\left(\mathrm{g} \mathrm{cm}^{-3}\right.$ de suelo seco).

Tratándose del agua (peso específico $=1$ ), 1 gramo equivale a $1 \mathrm{~mL}$.

El potencial hídrico se evaluó mediante el método del papel de filtro, siguiendo el procedimiento expuesto por Deka et al. (1995) para el papel de filtro tipo Whatman $n^{\circ}$ 42. Es un método de laboratorio simple y económico que permite obtener indirectamente la succión del suelo mediante la medición del contenido gravimétrico del papel en equilibrio, el que está relacionado a la succión del suelo a partir de una curva de calibración predeterminada. Los filtros más usados son Whatman 42 y Sleicher y Schuell 59 (SS 59). En un recipiente cerrado herméticamente, se colocó un papel de filtro en contacto con el suelo y se lo dejó durante diez días, tiempo suficiente para que alcancen el equilibrio el agua en los poros de la muestra, en los poros del papel y en el aire del interior del recipiente. Se determinó posteriormente la masa del papel de filtro y se infirió el potencial mátrico de la muestra a partir de una curva de calibración, la cual relaciona el potencial y el tenor de agua en el papel (ASTM 2003).

Mediante el análisis de imágenes satelitales MODIS (moderate-resolution imaging spectro radiometer) (compuestos de NDVI cada 16 días del período 2008-2011) con una resolución espacial de $250 \mathrm{~m}$, se obtuvo el índice de diferencia de vegetación normalizada (NDVI) (objetivo 2) relacionado a la fracción de la radiación solar fotosintéticamente activa interceptada, el cual puede considerarse un estimador de la productividad primaria neta aérea (PPNA) (Ruimy et al. 1994). Este índice aprovecha el comportamiento radiométrico característico de la vegetación en las bandas roja e infrarrojo cercano ya que los pigmentos foliares absorben en particular en la banda roja mientras que tienen una alta reflectancia en el infrarrojo cercano. En los mismos sitios donde se realizaron los muestreos de suelo y en un par adicional en la Selva de Mirtáceas se localizaron en campo doce puntos de referencia mediante un sistema de posicionamiento global (GPS) con una resolución espacial de $5 \mathrm{~m}$ : seis puntos correspondientes a bosques nativos y seis correspondientes a bosques monoespecíficos de ligustros (tres en Selva Basal y tres en Selva de Mirtáceas, respectivamente). Para evitar efectos de borde en los datos satelitales, se utilizaron los píxeles centrales de los parches excluyendo una franja periférica de $60 \mathrm{~m}$. Las imágenes fueron georeferenciadas (UTM zona $20 \mathrm{~S}$, DATUM WGS-84) y co-registradas mediante puntos de GPS para hacerlas espacialmente coherentes con los sitios de muestreo de campo. Una vez seleccionadas las áreas de interés se obtuvieron las variaciones temporales de NDVI como un índice de la actividad fotosintética y se reconstruyó la fenología de los diferentes bosques en el período mencionado.

Posteriormente se calcularon las diferencias relativas de NDVI en el período 2008-2011 para la Selva de Mirtáceas y la Selva Basal, tomando como referencia el bosque nativo, en donde

Dif Rel $=($ NDVI bosque de ligustro - NDVI bosque nativo)/NDVI bosque nativo

Adicionalmente, para analizar la relación entre el NDVI del bosque nativo y de ligustro y las distintas condiciones climáticas a lo largo del año, se calculó una versión modificada del índice de aridez de De Martonne para cada mes del período 2008-2010 [2]. El índice fue modificado para hacerlo consistente con los valores disponibles de precipitaciones y temperaturas reportados por una estación meteorológica cercana al área de muestreo perteneciente a la Estación Experimental Agroindustrial Obispo Colombres (este índice utiliza valores de temperaturas medias mientras que se disponía de valores de temperaturas máximas medias y mínimas medias a partir de las cuales se calculó un promedio). El índice aumenta bajo condiciones de balance hídrico más positivo, y se define como:

Índice de aridez de De Martonne (modificado) $=\mathrm{P} /(\mathrm{T}+10)$

Donde,

$\mathrm{P}=$ precipitación mensual $(\mathrm{mm})$.

$\mathrm{T}$ = promedio de la temperatura máxima y mínima media mensual $\left({ }^{\circ} \mathrm{C}\right)$. 
Posteriormente se analizó la relación entre las diferencias relativas de NDVI y el índice de aridez de De Martonne para el período 2008-2010.

Análisis de datos. Para analizar y describir la dinámica estacional de la humedad edáfica, potencial hídrico y NDVI, se agruparon los puntos de muestreo en bloques, correspondientes a pares de bosques dominados por ligustros y bosques nativos con similares condiciones de pendiente, exposición, altitud y edad sucesional. Se realizaron pruebas t para analizar las diferencias de medias entre los bosques por separado para cada mes del período de muestreo. Se realizó un análisis de regresión exponencial simple para estudiar la relación entre las diferencias relativas de NDVI y el índice de aridez de De Martonne. Los análisis se realizaron con el programa InfoStat 2009 (Di Rienzo et al. 2009).

\section{RESULTADOS}

El contenido volumétrico de agua edáfica fue variable a lo largo del año en ambos tipos de bosques, nativos e invadidos por ligustro, presentando diferencias entre la época seca (invierno-primavera) y la época húmeda (verano-otoño). Se observó una tendencia en la Selva de Mirtáceas a presentar mayor contenido de humedad que la Selva Basal, sin embargo, éstas diferencias no fueron estadísticamente significativas (figura 2A). En general, el bosque de ligustro mostró niveles menores de humedad edáfica durante todo
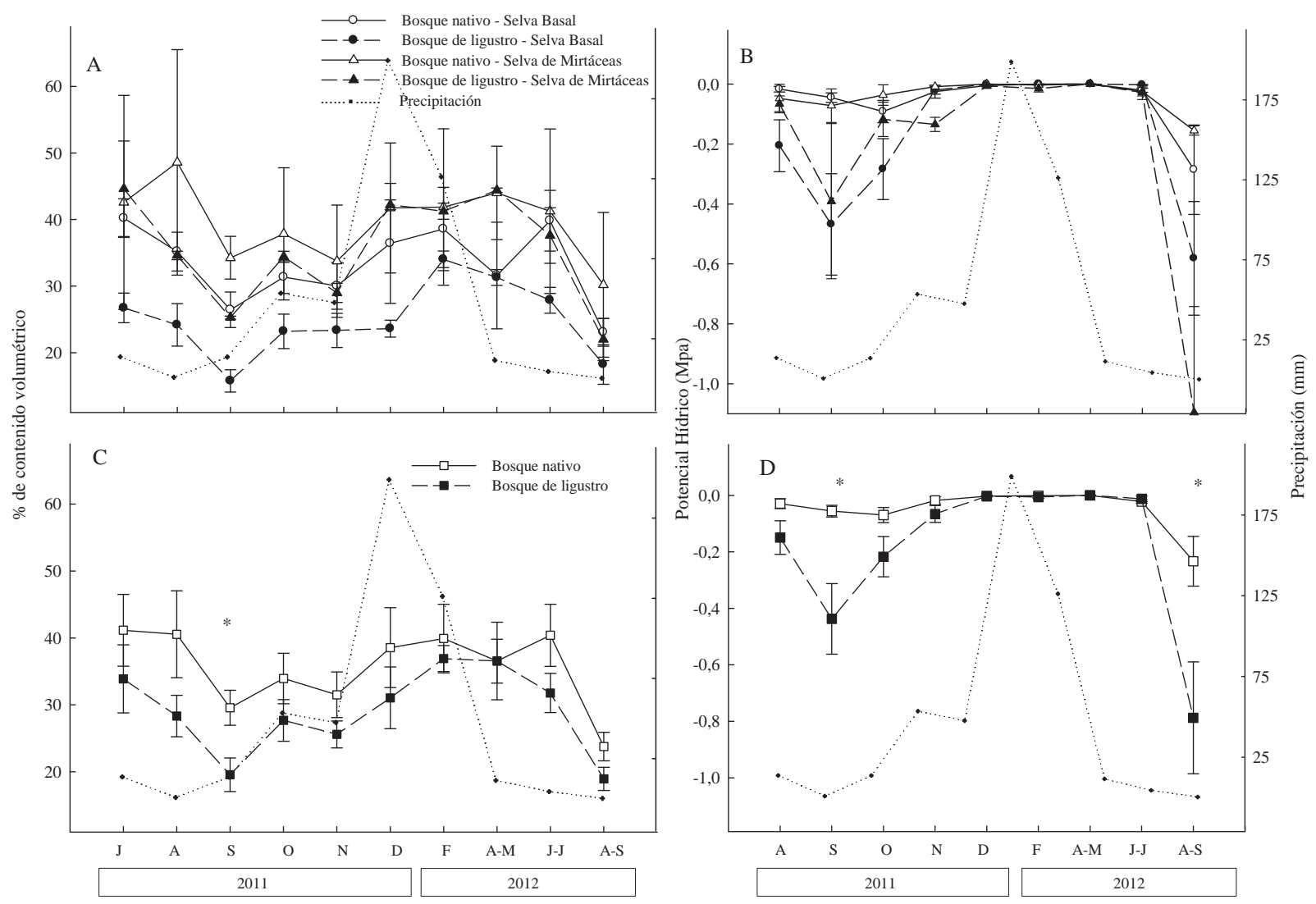

Figura 2. A) Variación en el porcentaje de humedad del suelo (contenido volumétrico) y B) del potencial hídrico a $25 \mathrm{~cm}$ de profundidad a lo largo del período julio 2011-setiembre 2012 en dos niveles altitudinales, Selva Basal y Selva de Mirtáceas. C) Variación del contenido volumétrico a $25 \mathrm{~cm}$ de profundidad en dos tipos de bosques, uno dominado por especies nativas (bosque nativo) y otro dominado por ligustro (bosque de ligustro). Se muestran las medias $\pm \mathrm{SE}, *$ diferencias estadísticamente significativas $(P<0,05)$. Prueba $\mathrm{t}=2,764 ; \mathrm{gl}=8 ; P=0,0245$. D) Variación del potencial hídrico del suelo a $25 \mathrm{~cm}$ de profundidad a lo largo del período agosto 2011-setiembre 2012 en dos tipos de bosques, uno dominado por especies nativas (bosque nativo) y otro dominado por ligustro (bosque de ligustro). Se muestran las medias $\pm \mathrm{SE}, *$ diferencias estadísticamente significativas $(P<0,05) . *$ Setiembre de 2011 prueba $\mathrm{t}=3,007$; gl = 8; $P=0,0169$; setiembre de 2012, prueba $\mathrm{t}=2,557$; $\mathrm{gl}=8 ; P=0,034$. La línea punteada muestra la variación anual de las precipitaciones.

A) Variation of soil moisture (volumetric water content) at $25 \mathrm{~cm}$ depth during the period July 2011 - September 2012; and B) water potential in two altitude levels, Basal Forest and Myrtaceae Forest. C) Change in volumetric water content in two forest types, one dominated by native

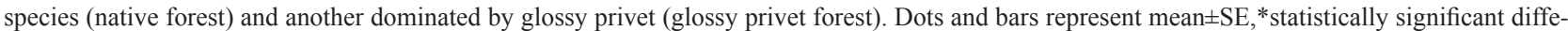
rence $(P<0.05)$. t-test $=2.764, \mathrm{df}=8, P=0.0245 . \mathrm{D})$. Variation of soil water potential at $25 \mathrm{~cm}$ depth during the period August 2011 - September 2012 in two forest types: one dominated by native species (native forest) and one dominated by glossy privet (glossy privet forest). Dots and bars represent mean \pm SE, ${ }^{*}$ statistically significant difference $(P<0.05)$. September 2011, t-test $=3.007, \mathrm{df}=8, P=0.0169$; September 2012, t-test $=2.557, \mathrm{df}=8$; $P=0.034$. Dotted line shows the annual variation in rainfall. 
el período de estudio. La mayor diferencia se dio en setiembre, mes de mayor déficit hídrico de la estación seca. En este mes, en 2011, los bosques dominados por ligustro presentaron 32,5\% menos humedad edáfica respecto de los nativos, y en setiembre de 2012 presentaron $24 \%$ menos. Al comienzo de la época húmeda (diciembre-febrero) el contenido volumétrico de agua en suelo de ambos bosques aumentó pero los ligustrales siguieron presentando un $13,4 \%$ menos de humedad (figura $2 \mathrm{C}$ ).

Las diferencias de potencial hídrico entre la Selva Basal y la Selva de Mirtáceas no presentaron un patrón estable durante los meses muestreados (figura 2B). El potencial hídrico del suelo fue notoriamente diferente entre ambos tipos de bosques durante la época seca, especialmente en el mes de mayor déficit hídrico (setiembre). En este mes, los ligustrales presentaron 0.38 MPa y 0.55 MPa menos que los bosques nativos, en los años 2011 y 2012 respectivamente. Es decir, un potencial hídrico ocho y cuatro veces menor respectivamente. En los meses de diciembre, enero y febrero (meses de mayor precipitación) los ligustrales presentaron 0,002 MPa menos que los bosques nativos; es decir, la mitad del potencial hídrico de los mismos (figura 2D).
En los valores de NDVI se observó una variación a lo largo del año durante el período analizado (2008-2011) en ambos tipos de bosques (nativo e invadido) en los dos niveles altitudinales estudiados (Selva Basal y Selva de Mirtáceas) correspondientes con la época seca (inviernoprimavera) y la época húmeda (verano-otoño). Los bosques de ligustro tendieron a presentar mayor NDVI respecto a los bosques nativos, particularmente en la época seca, aunque estas diferencias no fueron estadísticamente significativas (figura 3 ).

En cuanto a las diferencias relativas de NDVI entre bosques nativos y de ligustro, se observó que entre los bosques situados a mayor altura (correspondiente a la Selva de Mirtáceas, 1280-1450 m snm) la diferencia es menor que entre los bosques ubicados a menor altura (Selva Basal, 630-780 m snm) (figura 4). Durante la época seca, los bosques situados a menor altura presentaron diferencias relativas mayores que en la época húmeda, es decir que los valores medios de NDVI del bosque de ligustro fueron mayores que los del bosque nativo de Selva Basal, siendo la diferencia en el año 2008, de 11,5\%, en el 2009 de $15 \%$, en 2010 de 10,7 \% y en 2011 de $12 \%$. Por otro lado, entre
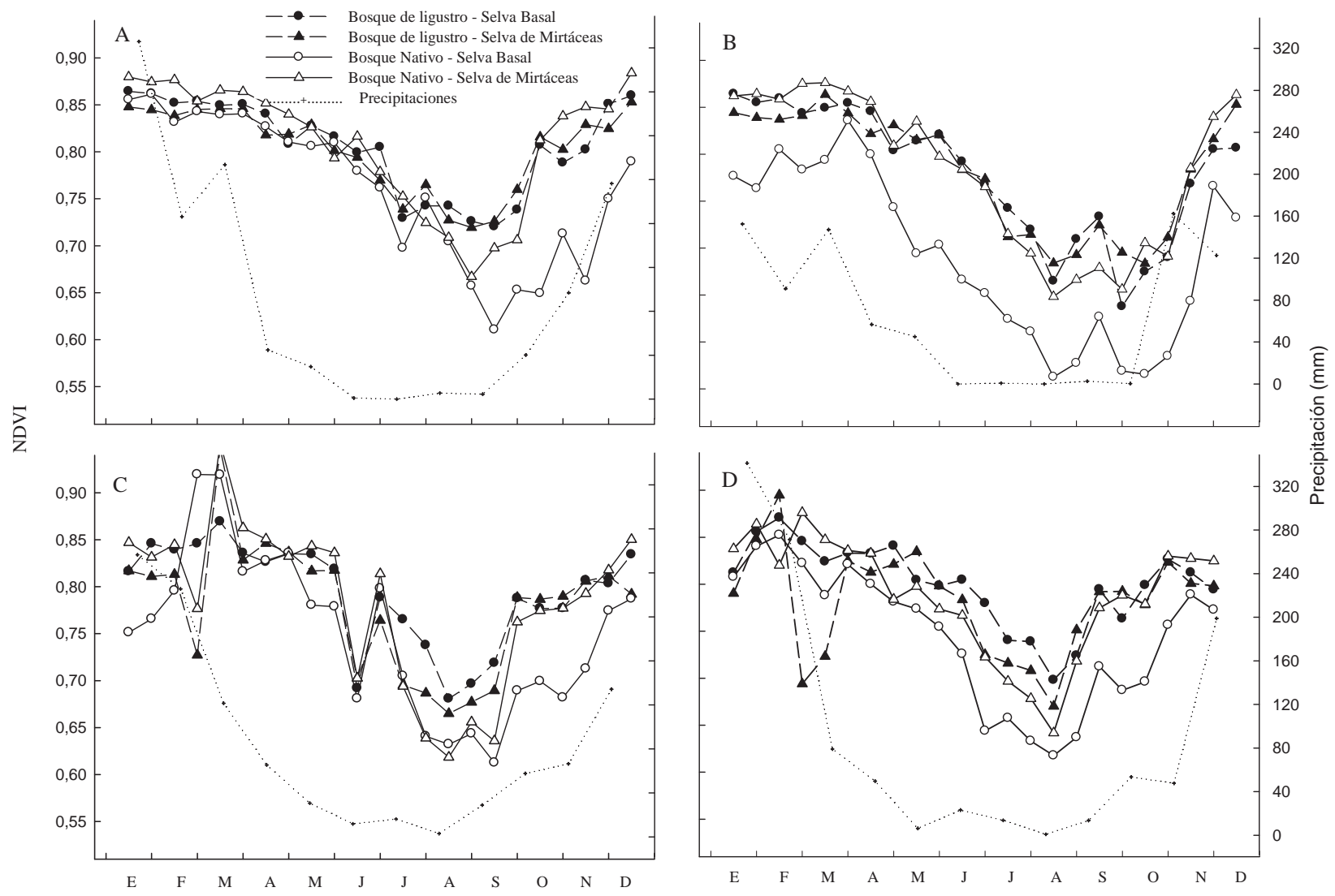

Figura 3. Variación del NDVI (medias) a lo largo del período A) 2008, B) 2009, C) 2010, D) 2011 en dos tipos de bosques, uno dominado por especies nativas (bosque nativo) y otro dominado por ligustro (bosque de ligustro), en dos niveles altitudinales, Selva Basal y Selva de Mirtáceas. La línea punteada muestra la variación anual de las precipitaciones.

Variation of NDVI (average) over the period A) 2008, B) 2009, C) 2010 and D) 2011 in two forest types, one dominated by native species (BN) and one dominated by glossy privet (BL); in two altitudinal levels, Basal Forest (SB) and Myrtaceae Forest (SM). Dotted line shows the annual variation in rainfall. 


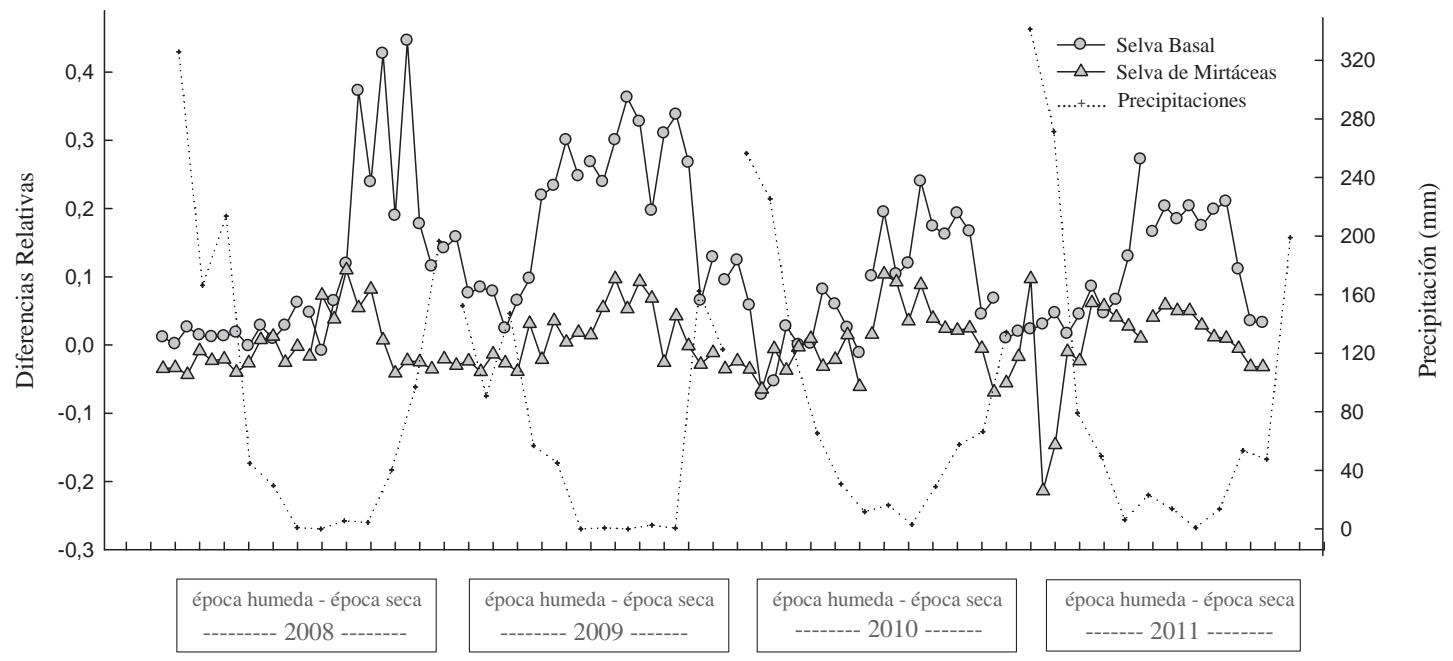

Figura 4. Diferencias relativas de NDVI entre bosques de ligustros y bosques nativos en la Selva Basal $(\bullet)$ y entre bosques de ligustros y bosques nativos en la Selva de Mirtáceas ( $\mathbf{\Delta}$ ), durante el período 2008-2011, tomando como referencia el bosque nativo. Dif Rel = (NDVI lig - NDVI nat)/NDVI nat. La línea punteada muestra la variación anual de las precipitaciones.

Relative differences of NDVI between glossy privet forests and native forests in the Myrtaceae Forest $(\boldsymbol{\Delta})$ and between glossy privet forests and native forests in the Basal Forest $(\bullet)$, during the period 2008-2011, with reference to the native forest. Rel Diff $=($ NDVI lig - NDVI nat) $/$ NDVI nat. Dotted line shows the annual variation in rainfall.

los bosques situados a mayor altura, en los períodos húmedos las diferencias relativas fueron negativas, es decir que los valores de NDVI del bosque de ligustro fueron menores que los del bosque nativo de la Selva de Mirtáceas y, durante los períodos secos, las diferencias relativas tendieron a ser positivas tomando los ligustrales valores de NDVI un poco mayores que los bosques nativos con diferencias en el año 2008 de 5,5 \%, en 2009 de 5,3 \%, en 2010 de 5,2 $\%$ y en 2011 de $3,7 \%$. A su vez, estos resultados permiten observar que las diferencias de NDVI anuales y, sobre todo en la época seca, entre bosques invadidos y nativos son menores en la Selva de Mirtáceas que en la Selva Basal.

Adicionalmente, se observó una relación negativa entre las diferencias relativas de NDVI de bosques nativos y ligustrales y el índice de aridez de De Martonne (figura 5). Esta fue estadísticamente significativa en ambos niveles altitudinales, en el año 2009 y cuando se consideró todos los años en conjunto. A medida que las condiciones climáticas se hacen más áridas (menor índice de De Martonne), las diferencias entre el NDVI de los ligustrales y de los bosques nativos son aproximadamente un $20 \%$ mayores, es decir, los ligustrales presentan mayores valores de NDVI que los bosques nativos. Las diferencias tienden a hacerse nulas en los meses de mayor humedad.

\section{DISCUSIÓN}

Existen casos en que la invasión de plantas exóticas altera el ciclo hidrológico provocando cambios en la tasa y el régimen de evapotranspiración e incluso en la escorrentía, debido a diferencias en las tasas de transpiración y en la fenología de las especies invasoras y nativas (Levine et al. 2003). Si bien las cuencas son sistemas complejos y los impactos sobre la dinámica hídrica deben ser considerados en conjunto, los resultados obtenidos en este trabajo permiten una primera aproximación a los efectos de la invasión por Ligustrum lucidum sobre algunos aspectos del ciclo hidrológico de las Yungas y sugieren que está influyendo sobre su dinámica hídrica.

El contenido de humedad edáfica es una característica importante para el desarrollo de la vegetación ya que garantiza la disponibilidad de agua, incluso, en épocas de bajas precipitaciones. Consistentemente con lo esperado, en este estudio se observó que la humedad edáfica a los $25 \mathrm{~cm}$ de profundidad es significativamente menor en los bosques invadidos por ligustro que en los bosques nativos, especialmente durante la estación más seca (invierno-primavera) y que el agua disponible a esta profundidad, expresada como potencial hídrico, disminuye abruptamente en estos bosques (figura 2). Estudios en zonas con regímenes de precipitaciones de tipo mediterráneo, demostraron que las especies forestales exóticas de rápido crecimiento, como Pinus spp. y Eucalyptus spp., consumen el agua disponible en mayor cantidad y de forma menos eficiente respecto a las especies nativas (Gyenge et al. 2011). Los resultados del presente trabajo indican que el ligustro estaría afectando el contenido de agua en suelo, al menos en los horizontes más superficiales y que podría limitar el acceso a este recurso por parte de otras especies. Licata et al. (2008), observaron que en la época seca en el noroeste de la Patagonia, más del $60 \%$ del agua utilizada, tanto por bosques de Pinus ponderosa (Dougl.) Laws (exótica) como por bosques nativos, provenía de más de $80 \mathrm{~cm}$ de profundidad mientras que, en la época húmeda, más del $60 \%$ provenía de la capa edáfica 


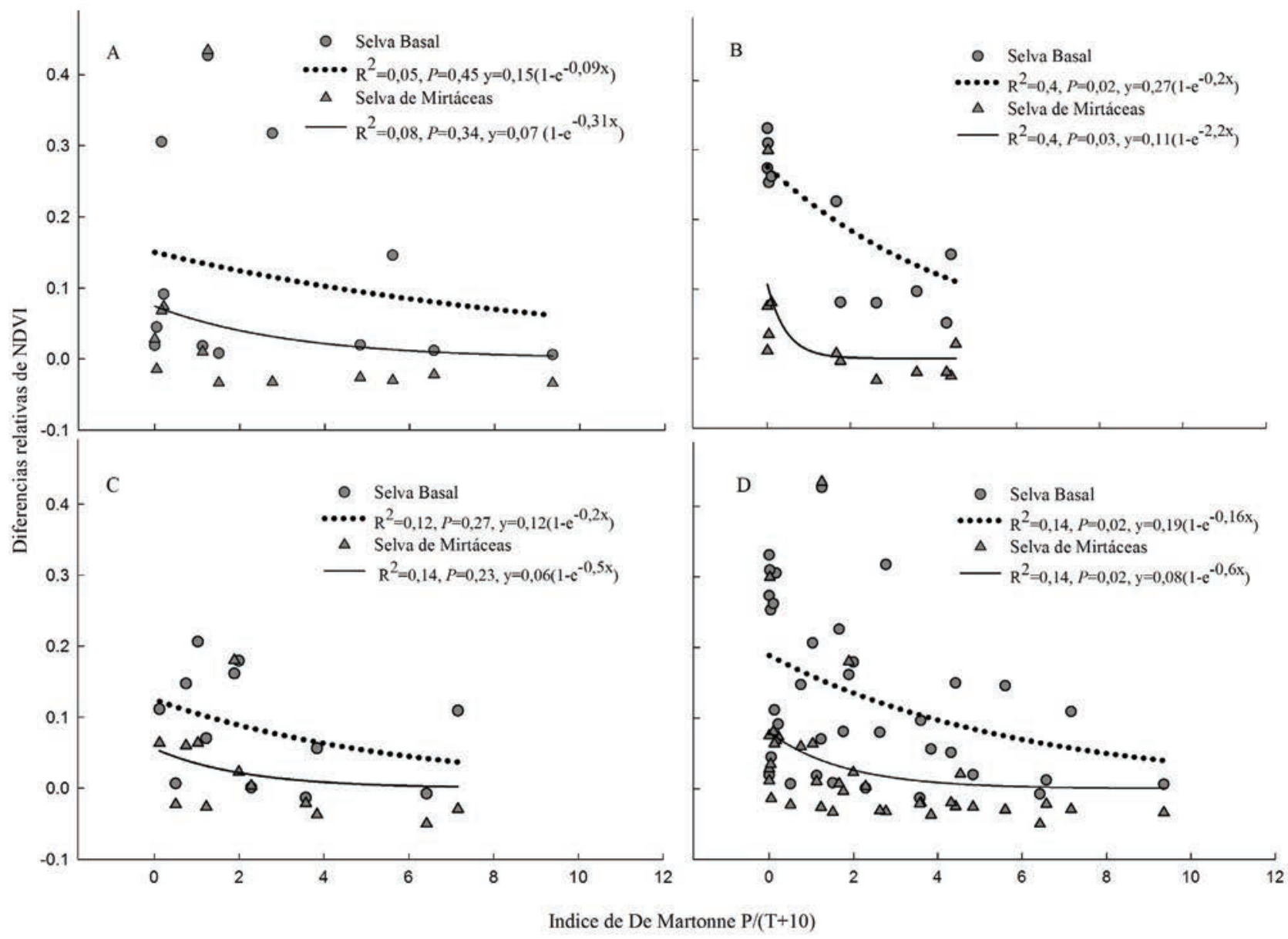

Figura 5. Relación entre las diferencias relativas de NDVI entre bosques de ligustro y bosque nativos en la Selva Basal (•) y en la Selva de Mirtáceas ( $\boldsymbol{\Delta}$ ) (tomando como referencia el bosque nativo) y el índice de aridez de De Martonne para los años A) 2008, B) 2009, C) 2010 y D) los tres años juntos. Las curvas representan la regresión exponencial.

Relationships between relative differences in NDVI of glossy privet forests and native forest in Basal Forest $(\bullet)$ and Forest of Myrtaceae ( $\Delta$ ) (with reference to the native forest) and the aridity index of De Martonne for A) 2008, B) 2009, C) 2010 and D) three years together. Strait lines represent exponential regression equations.

por encima de los $80 \mathrm{~cm}$. Es probable que la utilización y la dinámica del agua del suelo en el presente estudio sean más complejas que la propuesta por el modelo de las dos capas. Posiblemente el bosque nativo, más diverso que el de ligustro y con un gran número de especies con diferentes morfologías de sistemas radiculares, presente una distribución de las raíces a distintas profundidades del suelo y redistribución hidráulica mientras que los bosques monodominantes de ligustros no presenten estas adaptaciones provocando el bajo contenido de humedad encontrado en los primeros centímetros de profundidad del suelo. Para evaluar esta hipótesis serían necesarios muestreos de suelo a mayor profundidad, estudios de distribución radicular y su relación con la variación del contenido de humedad del perfil del suelo; así como también estudios que involucren isotopos estables y flujo de savia del xilema.

Por otro lado, el dosel podría jugar un papel importante en la intercepción de las precipitaciones. Iroumé y
Huber (2000) observaron que los bosques de Pseudotsuga menziesii (Mirb.) Franco (exótica) interceptan en mayor medida las precipitaciones en comparación con el bosque nativo debido a sus diferentes fenologías. En otro estudio, Iroumé y Huber (2002) obtuvieron similares resultados, pero observaron que los patrones de intercepción de las precipitaciones se relacionaban principalmente con las características de las mismas y las condiciones meteorológicas durante la etapa de crecimiento y dormición; y que el nivel de densidad y las características del dosel influyeron secundariamente. Una situación similar podría estar sucediendo en el presente trabajo, siendo probable que el dosel perennifolio del bosque de ligustro intercepte un mayor porcentaje de precipitaciones y que a su vez las variables climáticas influyan en el contenido edáfico de agua, como se observa cuando se comparan el nivel de Selva de Mirtáceas, más húmedo, con el de Selva Basal. Esta hipótesis sería concordante con la mayor cobertura y área basal en- 
contradas en los ligustrales con respecto al bosque nativo (Ayup et al. 2014). Otro aspecto importante a considerar es el análisis de estas tendencias y patrones en función de la escala. En este sentido, Cavaleri y Sack (2010), realizaron una revisión de trabajos en los que se compara el uso del agua entre plantas invasoras y nativas con igual forma de crecimiento a múltiples escalas: hoja, planta y ecosistemas. Estos autores detectaron diferencias notorias: a escala hoja, se encontró una fuerte tendencia a una mayor conductancia estomática en las especies invasoras que en las nativas. A escala planta, las diferencias entre el flujo diario de savia por área de albura entre pares de especies no fue significativa, pero sí hubo una tendencia significativa en las especies invasoras a tener menores tasas de flujo de savia diarias por unidad de área foliar. A escala ecosistema hubo muchos menos datos disponibles, sin embargo, observaron que, aquellos dominados por invasoras, tuvieron mayores tasas de flujo de savia diaria por unidad de superficie que los ecosistemas dominados por nativas.

Hoyos et al. (2010) registraron en los bosques serranos de la provincia de Córdoba que las áreas severamente invadidas por ligustro muestran altos valores de NDVI y cambios en la fenología con respecto a los bosque nativos. El presente estudio es parcialmente consistente con la predicción planteada a partir del segundo objetivo, que los ligustrales tienen mayor NDVI, principalmente durante la época seca, sin embargo, estas diferencias no resultaron estadísticamente significativas (figuras 3 y 4). Esto puede deberse a que en las Yungas, en el período agosto-octubre (época seca) el bosque nativo presenta menor cantidad de follaje, y en diciembre-febrero (época húmeda) recupera gran parte del mismo, estabilizándose y manteniéndose así hasta el mes de junio, sufriendo una marcada caída nuevamente en julio (Boletta et al. 1995). El ligustro, en cambio, no pierde sus hojas en ninguna época del año (Grau y Aragón 2000). A pesar de las diferencias de NDVI observadas en la época seca entre ambos bosques, los resultados de este trabajo muestran en ambos una caída del NDVI durante esta época. Esto demuestra que durante el invierno la actividad fotosintética del ligustro disminuye en coincidencia con la menor disponibilidad hídrica y menor temperatura que también afecta al resto de las especies vegetales. Por otro lado, al perder las hojas los árboles nativos durante el invierno, se incrementa la llegada de luz al suelo. La disponibilidad de luz es uno de los mayores determinantes en la economía de carbono de las plantas y por lo tanto juega un papel fundamental en la productividad (Reich et al. 1992). El sotobosque del bosque nativo, que es mucho más diverso y denso que el del bosque invadido (Ayup et al. 2014) tendría un porcentaje de especies que aprovechan esta mayor disponibilidad de luz para crecer durante esta época y aportar significativamente al incremento de los valores de NDVI registrado. Mientras que los bosques de ligustro, al no perder las hojas en ninguna estación, aprovecharían la luz a lo largo de todo el año, explicando también las mayores tasas de crecimiento encontradas con respecto a los árboles nativos (Easdale et al. 2007) e imposibilitando el uso de este recurso al sotobosque mucho menos diverso de estos bosques. Esta situación acotaría las diferencias en los valores de NDVI observadas a lo largo del año entre los dos tipos de bosques. Estudios fisiológicos y de campo son necesarios a la hora de corroborar estas hipótesis.

Según Ewe y Sternberg (2006) y Fritzsche et al. (2006), algunas especies exóticas invasoras con capacidad de alterar el uso del agua (e.g. Schinus spp. y Eucalyptus spp.) están menos sujetas a las fluctuaciones climáticas estacionales en contraste con los bosques nativos. En este estudio se observó, por un lado, que los valores de NDVI de ambos bosques están influenciados por las condiciones climáticas estacionales (reflejadas en el índice de aridez de De Martonne). Sin embargo, las diferencias relativas entre el bosque de ligustro y el bosque nativo se acentúan durante la época seca, indicando que el bosque nativo es más sensible a las fluctuaciones climáticas (figura 5). Esto permitiría suponer que, ante sequías intensas, la productividad del ligustro sería menos afectada que la del bosque nativo. Por otro lado, se sabe que la Selva de Mirtáceas tiene menor estacionalidad hídrica que la Selva Basal, mayores precipitaciones y aportes adicionales de humedad debido a la presencia de neblinas (Hunzinger 1997). Esto explica por qué, al analizar los datos teniendo en cuenta los dos niveles altitudinales estudiados, se observó que la Selva de Mirtáceas tendió a presentar valores mayores de humedad edáfica (figura 2) y de NDVI (figura 3) y menores diferencias relativas de NDVI entre ambos tipos de bosques (figura 4). A pesar de esta variación en las diferencias entre bosques a distintas alturas, se observó un menor contenido de humedad edáfica en los ligustrales en comparación con los bosques nativos, durante la época seca; posiblemente por una mayor actividad fisiológica (e.g. mayor fotosíntesis) durante esta época respecto al bosque nativo. Esto sugiere a su vez que la invasión del ligustro podría tener mayores efectos hidrológicos en los sectores más áridos de las Yungas (como en el área periurbana de Salta y Jujuy) y en otros ecosistemas caracterizados por una menor disponibilidad hídrica, como zonas de Chaco serrano en las sierras de Córdoba.

Para una mejor evaluación de los aspectos aquí descritos, son necesarios futuros estudios sobre la evapotranspiración, la intercepción de las precipitaciones, el contenido de humedad edáfica a mayor profundidad y las características ecofisiológicas de la especie (fotosíntesis, arquitectura hidráulica, traslocación de fotoasimilados, etc.). Estos podrían brindar un aporte más completo sobre el uso del agua por parte de esta especie invasora que está reemplazando los bosques nativos de las Yungas e impactando sobre la dinámica hídrica de este ecosistema.

\section{CONCLUSIONES}

El presente estudio aporta evidencias sobre la manera en que la invasión de ligustro en las Yungas puede alterar algunos aspectos de su funcionamiento hídrico, lo que se 
refleja en un menor contenido de agua en suelo. Este efecto es más marcado en la Selva Basal, localizada a menor altura y con mayor déficit hídrico inverno-primaveral, que en la Selva de Mirtáceas. Es también mucho más contrastante en la estación de mayor déficit hídrico. La combinación de situaciones de topografía, patrones climáticos, geografía humana y dinámica de la vegetación del área de estudio (piedemonte en la interfase natural urbana con fuerte incidencia de invasión por ligustro) es representativa también de otras situaciones del noroeste argentino (Salta, San Salvador de Jujuy) y, en menor medida, de ciudades del piedemonte de la provincia de Córdoba, por lo que los datos de este estudio son también relevantes a escalas geográficas más extensas.

\section{AGRADECIMIENTOS}

Agradecemos a Marta Ayup, Roxana Aragón, Nicolás Brandán y Paula Boggiatto, quienes colaboraron en el trabajo de campo y discusión de los resultados. Dos revisores anónimos enriquecieron el artículo con valiosos aportes sobre métodos y conceptos. Estos estudios fueron financiados parcialmente por Rufford Foundation, CONICET, CIUNT y PICT 1382.

Agradecemos a la Estación Experimental Agroindustrial Obispo Colombres por los datos climáticos brindados.

\section{REFERENCIAS}

Aragón R, JM Morales. 2003. Species composition and invasion in secondary forest of northwestern Argentina affected by land use history, environmental conditions and landscape context. Journal of Vegetation Sciences 14: 195-204.

Aragón R, M Groom. 2003. Invasion by Ligustrum lucidum in NW Argentina: plant characteristics in different habitat types. Revista de Biología Tropical 51: 59-70.

ASTM (American Society For Testing And Materials, US). 2003. Standard test method for measurement of soil potential (suction) using filter paper, D5298-03. Philadelphia, USA. 6 p.

Ayup MM, LF Montti, R Aragón, HR Grau. 2014. Invasion of Ligustrum lucidum (Oleaceae) in the Southern Yungas. Changes in habitat properties and decline in bird diversity. Acta Oecologica 54: 72-81.

Boletta PE, R Vides-Almonacid, RE Figueroa, MT Fernández. 1995. Cambios fenológicos de la selva basal de Yungas en Sierra de San Javier (Tucumán, Argentina) y su relación con la organización estacional de las comunidades de aves. In Brown AD y HR Grau eds. Investigación, conservación y desarrollo de selvas subtropicales de montaña. Proyecto de desarrollo agroforestal LIEY. Tucumán, Argentina. p 103-114.

Brown A. D. 2009. Las selvas pedemontanas de las Yungas Manejo sustentable y conservación de la biodiversidad de un ecosistema prioritario del noroeste argentino. In Brown AD, PG Blendinger, T Lomáscolo, P García eds. Selva Pedemontana de las Yungas. Historia natural, ecología y manejo de un ecosistema en peligro. Buenos Aires, Argentina. Ediciones del Subtrópico. p 13-36.

Campanello PI. 2004. Diversidad, crecimiento y fisiología de árboles en la Selva Misionera: efectos de los cambios en la estructura y funcionamiento del ecosistema producidos por la invasión de lianas y bambúseas. Tesis doctoral. Universidad Nacional de Buenos Aires. 164 p.

Casavecchia MC. 2004. Aplicaciones de imágenes Landsat para detección y monitoreo de bosques de siempreverde (Ligustrum lucidum) en la sierra de San Javier, Tucumán, Argentina. Tesis de grado. Tucumán, Argentina. Facultad de Ciencias Naturales, Universidad Nacional de Tucumán. 51 p.

Cavaleri MA, L Sack. 2010. Comparative water use of native and invasive plants at multiple scales: a global meta-analysis. Ecology 91(9): 2705-2715.

Cronk QCB, JL Fuller. 1995. Plant invaders: the threat to natural ecosystems. London, UK. Chapman and Hall. 241 p.

Deka RN, M Wairiu, PW Mtakwa, CE Mullins, EM Veenendaal, J Townsend. 1995. Use and accuracy of the filter-paper technique for measurement of soil matric potential. European Journal of Soil Science 46: 233-238.

Di Rienzo JA, F Casanoves, MG Balzarini, L González, M Tablada, CW Robledo. 2009. InfoStat versión 2009. Grupo InfoStat, FCA, Universidad Nacional de Córdoba, Argentina.

Easdale TA, J Healey, HR Grau, A Malizia. 2007. Tree life histories in montane subtropical forests: species differ independently by shade tolerance, turnover rate and substrate preference. Journal of Ecology 95: 1234-1249.

Eremchuk JE, J Martensen. 1996. Geología ambiental del municipio de Yerba Buena (Provincia de Tucumán, Argentina). In Actas XIII Congreso Geológico Argentino y III Congreso de Exploración de Hidrocarburos. Buenos Aires, Argentina. Volumen 4: 445-462.

Ewe SML, LSL Sternberg. 2006. Water uptake patterns of an invasive exotic plant in coastal saline habitats. Journal of Coastal Research 23(1): 255-264.

Fritzsche F, A Abate, M Fetene, E Beck, S Weise, G Guggenberger. 2006. Soil-plant hydrology of indigenous and exotic trees in an Ethiopian montane forest. Tree Physiology 26: 1043-1054.

Gavier-Pizarro GI, T Kuemmerle, LE Hoyos, SI Stewart, CD Huebner, NS Keuler, VC Radeloff. 2012. Monitoring the invasion of an exotic tree (Ligustrum lucidum) from 1983 to 2006 with Landsat satellite data and a support vector machine in Córdoba, Argentina. Remote Sensing of Environment 122: 134-145.

Grau HR, R Aragón. 2000. Árboles Invasores de la Sierra de San Javier, Tucumán Argentina. In Grau HR, R Aragón eds. Ecología de árboles exóticos en las yungas Argentinas. Tucumán. Argentina. LIEY. p 5-20.

Grau HR, ME Hernández, J Gutierrez, NI Gasparri, MC Casavecchia, EE Flores, L Paolini. 2008. A peri-urban neotropical forest transition and its consequences for environmental services. Ecology and Society 13(1): 35.

Gyenge JE, ME Fernández, J Licata, M Weigandt, BJ Bond, TM Schlichter. 2011. Uso del agua y productividad de los bosques nativos e implantados en el NO de la Patagonia: aproximaciones desde la ecohidrología y la ecofisiología. Ecología Austral 21: 271-284.

Hoyos LE, GI Gavier-Pizarro, T Kuemmerle, EH Bucher, VC Radeloff, PA Tecco. 2010. Invasion of glossy privet (Ligustrum lucidum) and native forest loss in the Sierras Chicas of Córdoba, Argentina. Biological Invasions 12: 3261-3275.

Hunzinger H. 1997. Hydrology of montane forests in the Sierra de San Javier, Tucumán, Argentina. Mountain Research 
and Development 17: 299-308.

Instituto de Botánica Darwinion, Herbario. Consultado 3 dic. 2013 Disponible en http://www2.darwin.edu.ar/Herbario/ Bases/ResultadosCtaIris.asp

Iroumé A, A Huber. 2000. Intercepción de las lluvias por la cubierta de bosques y efecto en los caudales de crecida en una cuenca experimental en Malalcahuello, IX Región, Chile. Bosque 21(1): 45-56.

Iroumé A, A Huber. 2002. Comparison of interception losses in a broadleaved native forest and a Pseudotsuga menziesii (Douglas fir) plantation in the Andes Mountains of southern Chile. Hydrological Processes 16: 2347-2361.

Le Maitre DC, BW Van Wilgen, RA Chapman, DH McKelly. 1996. Invasive plants and water resources in the Western Cape Province, South Africa: modelling the consequences of a lack of management. Journal of Applied Ecology 33: 161-172.

Levine JM, M Vilà, CM D’Antonio, JS Dukes, K Grigulis, S Lavorel. 2003. Mechanisms underlying the impact of exotic plant invasions. Philosophical Transactions of the Royal Society of London B 270: 775-781.

Licata JA, JE Gyenge, ME Fernández, TM Schlichter, BJ Bond. 2008. Increased water use by ponderosa pine plantations in northwestern Patagonia, Argentina compared with native forest vegetation. Forest Ecology and Management 255: 753-764.

Lichstein JW, HR Grau, R Aragón. 2004. Recruitment limitation in secondary forests dominated by an exotic tree. Journal of Vegetation Science 15: 721-728.

Mack RN, D Simberloff, WM Lonsdale, WM Evans, M Clout, FA Bazzaz. 2000. Biotic invasions: causes, epidemiology, global consequences, and control. Ecological Applications 10: 689-710.

Mark AD. 2009. Invasion biology. New York, USA. Oxford University Press. $258 \mathrm{p}$.

Montaldo NH. 2000. Reproductive success of bird-dispersed plants in a subtropical forest relict in Argentina. Revista Chilena de Historia Natural 73: 511-524.

Mooney HA, RJ Hobbs. 2000. Invasive Species in a Changing World. Island Press. $461 \mathrm{p}$.

Nosetto MD, EG Jobbágy, AB Brizuela, RB Jackson. 2011. The hydrologic consequences of land cover change in central Argentina. Agriculture, Ecosystems \& Environment 154: 2-11.
Panetta FD. 2000. Fates of fruits and seeds of Ligustrum lucidum W.T. Ait. and L. sinense Lour. maintained under natural rainfall or irrigation. Australian Journal of Botany 48(6):701-706.

Paula Lima W. 2010. A silvicultura e a Água. Ciência, dogmas, desafios. Cadernos do Diálogo - Rio de Janeiro, Brasil. Instituto Bio Atlântica. 65 p.

Reich PB, MB Walters, DS Ellsworth. 1992. Leaf Life-Span in Relation to Leaf, Plant, and Stand Characteristics among Diverse Ecosystems. Ecological Monographs 62: 365-392.

Río ME, L Archával. 1904. Geografía de la provincia de Córdoba: Atlas. Vol. 1. Publicación Oficial, Córdoba. Compañía Sud-americana de Billetes de Banco.

Ruimy A, B Saugier, G Dedieu. 1994. Methodology for the estimation of terrestrial net primary production from remotely sensed data. Journal of Geophysical Research 99: 52635283.

Sesma PJ, EY Guido, M E Puchulu. 2010. Geología de la ladera oriental de la sierra de San Javier, soporte físico para la gestión territorial. In Grau HR ed. Ecología de una interfase natural-urbana. La sierra de San Javier y el Gran San Miguel de Tucumán. San Miguel de Tucumán, Argentina. Editorial de la Universidad Nacional de Tucumán. p. 19-32.

Shafroth PB, JR Cleverly, TL Dudley, JP Taylor, C Van Riper III, EP Weeks, JN Stuart. 2005. Control of Tamarix spp. in the western U.S.: implications for water salvage, wildlife use, and riparian restoration. Environmental Management 35: 231-246.

Silveira L, J Alonso, L Martínez. 2006. Efecto de las plantaciones forestales sobre el recurso agua en el Uruguay. Agrociencia 10(2): 75-93.

Tecco PA, S Diaz, M Cabido, C Urcelay. 2010. Functional traits of alien plants across contrasting climatic and land-use regimes: do aliens join the locals or try harder than them? Journal of Ecology 98: 17-27.

Tineo A, E D’Urso, G Galindo, MM Fernández. 1985. Características Hidrogeológicas del borde oriental de la Sierra de San Javier. Provincia de Tucumán, República Argentina. In Actas del IV Congreso Geológico Chileno. Universidad del Norte, Antofagasta. p 5-8.

Zuccardi RB, GS Fadda, RA Benavidez. 1967. Relevamiento Edafológico en los Departamentos Tafí y Capital, Provincia de Tucumán. Revista Agronómica del Noroeste Argentino 6(1-2): 5-71. 
